\title{
RESEARCH
}

Open Access

\section{Recruitment and retention of participant and study partner dyads in two multinational Alzheimer's disease registration trials}

\author{
Olivia M. Bernstein ${ }^{1 *}$ D, Joshua D. Grill ${ }^{2,3,4,5+}$ and Daniel L. Gillen ${ }^{1,2,3+}$
}

\begin{abstract}
Background: Early study exit is detrimental to statistical power and increases the risk for bias in Alzheimer's disease clinical trials. Previous analyses in early phase academic trials demonstrated associations between rates of trial incompletion and participants' study partner type, with participants enrolling with non-spouse study partners being at greater risk.

Methods: We conducted secondary analyses of two multinational phase III trials of semagacestat, an oral gamma secretase inhibitor, for mild-to-moderate AD dementia.

Cox's proportional hazards regression model was used to estimate the relationship between study partner type and the risk of early exit from the trial after adjustment for a priori identified potential confounding factors. Additionally, we used a random forest model to identify top predictors of dropout.

Results: Among participants with spousal, adult child, and other study partners, respectively, 35\%, 38\%, and 36\% dropped out or died prior to protocol-defined study completion, respectively. In unadjusted models, the risk of trial incompletion differed by study partner type (unadjusted $p$ value $=0.027$ for test of differences by partner type), but in models adjusting for potential confounding factors, the differences were not statistically significant ( $p$ value $=$ 0.928). In exploratory modeling, participant age was identified as the primary characteristic to explain the relationship between study partner type and the risk of failing to complete the trial. Participant age was also the strongest predictor of trial incompletion in the random forest model.

Conclusions: After adjustment for age, no differences in the risk of incompletion were observed when comparing participants with different study partner types in these trials. Differences between our findings and the findings of previous studies may be explained by differences in trial phase, size, geographic regions, or the composition of academic and non-academic sites.
\end{abstract}

Keywords: Alzheimer's disease, Clinical trials, Recruitment, Retention, Study partner

\footnotetext{
* Correspondence: obernste@uci.edu

†'Joshua D. Grill and Daniel L. Gillen are co-senior authors.

'Department of Statistics, University of California, Irvine, Bren Hall 2019, Irvine, CA 92697-1250, USA

Full list of author information is available at the end of the article
}

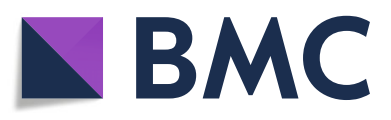

(c) The Author(s). 2021 Open Access This article is licensed under a Creative Commons Attribution 4.0 International License, which permits use, sharing, adaptation, distribution and reproduction in any medium or format, as long as you give appropriate credit to the original author(s) and the source, provide a link to the Creative Commons licence, and indicate if changes were made. The images or other third party material in this article are included in the article's Creative Commons licence, unless indicated otherwise in a credit line to the material. If material is not included in the article's Creative Commons licence and your intended use is not permitted by statutory regulation or exceeds the permitted use, you will need to obtain permission directly from the copyright holder. To view a copy of this licence, visit http://creativecommons.org/licenses/by/4.0/ The Creative Commons Public Domain Dedication waiver (http://creativecommons.org/publicdomain/zero/1.0/) applies to the data made available in this article, unless otherwise stated in a credit line to the data. 


\section{Background}

Clinical trials are essential for evaluating the safety and efficacy of potential disease-modifying drugs for Alzheimer's disease $(\mathrm{AD})$ but face notable challenges $[1,2]$. In particular, inefficient recruitment and challenging retention consistently delay AD trials and threaten their integrity. Barriers to recruitment include low awareness of trials, primary care physicians' reluctance to refer patients, participants' hesitancy to take investigational therapies and undergo invasive procedures, and strict inclusion criteria that may preclude participation for a large proportion of $\mathrm{AD}$ patients [3-5]. Resultantly, $\mathrm{AD}$ trial participants tend to be disproportionately white and with more years of education, compared to all $\mathrm{AD}$ patients in the USA [4-6].

All AD clinical trials require participants to enroll with a study partner. The study partner is often the participant's primary caregiver [5]. Study partners are integral to $\mathrm{AD}$ clinical trial conduct-they may assist with informed consent, ensure protocol compliance, and serve as informants for cognitive, functional, and behavioral outcome measures. Most primary caregivers for people with dementia are non-spouses, in particular adult children of the person with dementia [7-9]. Adult children are more likely than spouses to be working, caring for families, and have other responsibilities besides caregiving. Thus, it may be more difficult for adult children to fulfill trial obligations in long-term studies [5].

We previously observed an association between study partner type and trial recruitment and retention in a meta-dataset composed of six trials funded by the National Institute of Aging (NIA) that enrolled participants with possible or probable AD. In these studies, $67 \%$ of patients enrolled with a spouse, $26 \%$ enrolled with an adult child study partner, and $7 \%$ enrolled with a study partner who was neither a spouse nor an adult child (herein "other"). Trial incompletion was higher among participants enrolling with a non-spouse study partner [10].

Early study exit due to dropout or death in clinical trials causes missing data. In the best case scenario, this results in a loss of statistical precision for estimated treatment effects and reduced power. In the worst case scenario, missing data can produce bias in the estimated treatment effect. Expert guidance on statistical handling of missing data is clear: the first priority should be to prevent its occurrence [11]. Understanding predictors of early study exit will allow trial investigators to better design trials and to identify and support participants at increased risk for incompletion in order to prevent missing data.

Whether the relationship between $\mathrm{AD}$ study partner type and trial completion is homogenous across varying trial types and designs is unknown. The phases of the drug development process have unique objectives and therefore unique study designs [12]. Additionally, site networks conducting industry-funded trials differ from academic trials and may enroll different patient populations. For example, NIA-funded mild cognitive impairment (MCI) trials may enroll larger proportions of apolipoprotein E \&4 (APOE4) carriers than do industryfunded trials [13]. An analysis of a single MCI trial found that academic sites had lower rates of dropout compared to commercial sites [14]. Alternatively, a recent review of an antidepressant clinical trial found no significant difference in dropout between academic and non-academic sites [15]. Multinational trials are essential to regulatory goals and a thorough understanding of drug safety, but may carry increased risk of variability, including differences in the proportions of enrolled study partner dyad types by geographic region $[16,17]$. Completion rates also may differ among global geographic regions in multinational trials [16].

The objective of this study was to quantify the relationship between study partner type and trial recruitment and retention in two multisite, industry-funded multinational registrational mild-to-moderate $\mathrm{AD}$ dementia trials and to assess whether previously found results in NIA-sponsored trials were replicated. We hypothesized that non-spousal dyads would be at higher risk for dropping out.

\section{Study methods \\ Data source}

We performed secondary data analyses of two multinational studies of semagacestat, an oral gamma secretase inhibitor, in mild-to-moderate $\mathrm{AD}$ dementia. The trials employed essentially identical inclusion criteria and protocols (ClinicalTrials.gov numbers: NCT00594568 and NCT00762411). Each trial enrolled subjects 55 years and older who met NINCDS-ADRDA diagnostic criteria for $\mathrm{AD}$ and had Mini-Mental State Examination (MMSE) scores between 16 and 26. The co-primary outcomes of the trials were the 76-week changes from baseline in Alzheimer's Disease Assessment Scale cognitive subscale (ADAS-cog) and Alzheimer's Disease Cooperative StudyActivities of Daily Living (ADCS-ADL) scale. ADAS-cog and ADCS-ADL scores were collected throughout the study and at visit 17 scheduled at week 76 (Fig. 1). Based upon a recommendation from the data and safety monitoring board (DSMB), the trials were stopped early for futility in August 2010 [18, 19]. Of 2648 randomized participants, 640 completed the primary endpoint. Among the remaining participants, 45 died, 908 were lost to follow-up, 2 screen failed, and 1053 were unable to complete due to sponsor decision.

The research protocols were approved by the Internal Review Board at each trial site, and written informed consent was collected from each participant or a 


\section{Protocol Schedule}

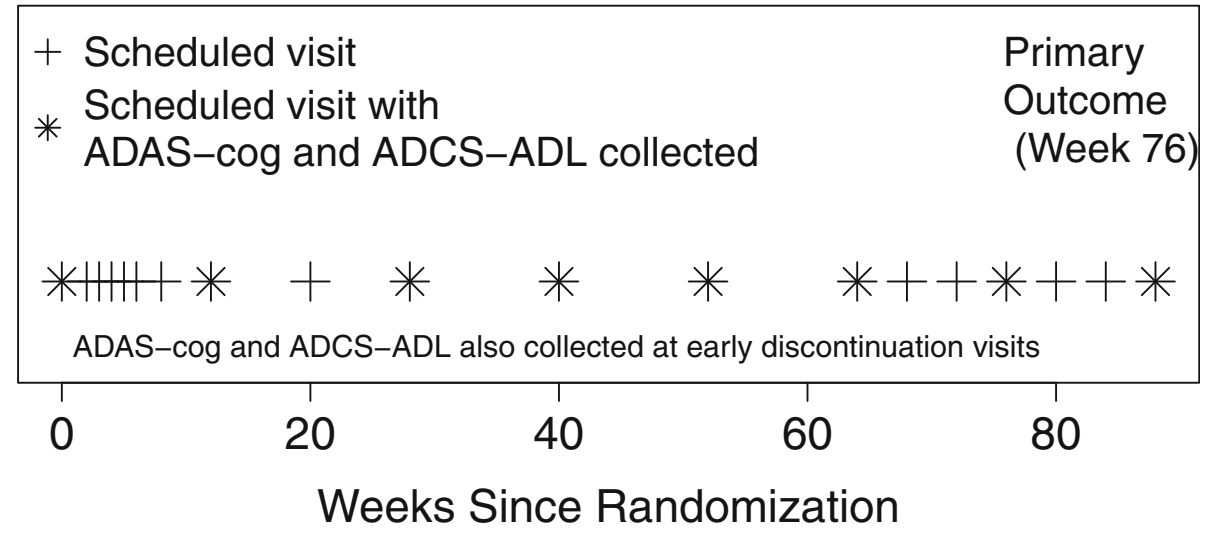

Fig. 1 Protocol schedule. Alzheimer's Disease Assessment Scale for cognition (ADAS-cog) and Alzheimer's Disease Cooperative Study-Activities of Daily Living (ADCS-ADL) were scheduled to be collected at 0, 12, 28, 40, 52, 64, 76, and 88 weeks and at any early study discontinuation visits. The primary outcome was collected at week 76

surrogate. The current study did not access participant identifying information and therefore does not meet the criteria for human subjects research.

\section{Data analysis/statistical methods}

Given the similarities between the protocols, we combined the two trials for analysis. We removed two subjects who reported a race of "other," 41 subjects with missing covariate values, and two subjects who screen failed. Of the two subjects with a race of "other," one was lost to follow-up and one completed the co-primary outcomes. Among the 41 subjects with missing covariates, 24 subjects were lost to follow-up, 12 had to withdraw from the study early due to the sponsor decision, and 5 completed the co-primary outcomes. Most of the missing data was confined to study partner characteristics and the Geriatric Depression Scale score. The final dataset used in this analysis included $N=2603$ participants. We used the baseline Resource Utilization in Dementia (RUD) to classify participants based on the type of study partner with whom they enrolled. We categorized the nations in which participants enrolled into seven global regions, as done previously [16, 20]. The seven regions included North America, South America/ Mexico, Eastern Europe/Russia, Western Europe/Israel, Australia/South Africa, Asia, and Japan. Descriptive statistics were stratified by study partner type and reported as mean (standard deviation) for quantitative variables and frequency (percent) for categorical variables unless otherwise stated.

We investigated the relationship between study partner type and retention by quantifying the time to early study exit using survival analysis. The outcome of our analysis was defined as the time from randomization to the first of (1) early study exit prior to completing the week 76 primary endpoint due to participant decision $(N=883)$ or $(2)$ death $(N=45)$. Among the 45 subjects who discontinued due to death, we observed stretches of time of up to 4 months between final visit dates and death dates. Out of concern that subjects had decided to drop out prior to their death or that worsening health prevented them from attending visits, we classified these subjects as dropping out at their final in-person visit. Subjects with ADAS-cog and ADCS-ADL scores reported at visit 17 or on visit 16 or 18 and within weeks 74 to 78 were considered to have completed the trial and were censored at their week 76 visit. Participants who discontinued because of the sponsor's decision to terminate the study based on the recommendation of the DSMB were censored at the time of study termination $(N=1041)$.

In the primary analysis, we used Cox's proportional hazards regression model to estimate the relative risk (RR) for early study discontinuation associated with study partner type. We used a partial likelihood ratio test (LRT) to assess if the risk of early exit differed by study partner type. To account for potential confounding, we a priori decided to adjust for participant characteristics of sex, age, education, APOE4 carrier status, global region, baseline cognitive functioning (MMSE), potential vascular contributions to cognitive impairment (Hachinski Ischemic Score), and depression (Geriatric Depression Scale), as well as study partner sex, study partner age, and study partner employment status. We did not adjust for race and ethnicity or the proportion of time the study partner and participant spend together because of concerns about lack of model identifiability due to multi-collinearity with global region and study partner type, respectively. To assess the robustness of our results to the modeling decisions, we performed two 
sensitivity analyses. First, we considered early study exit due to death as a censoring event instead of an observed exit time. Second, we restricted our analysis to participants in North America and re-ran the primary analysis while adjusting for race and ethnicity.

In exploratory analyses, we investigated the extent to which individual covariates could explain the relationship between study partner type and dropout. We compared the unadjusted model, fully adjusted model, and models that only adjusted for one potential confounder at a time to determine if the study partner effect was primarily attenuated by a single confounding factor. Finally, we ran a random forest model for survival data with logrank splitting to identify the top predictors of dropout with the highest variable importance values [21-23]. All analyses were performed in $\mathrm{R}$ version 3.6.0 [24].

\section{Results}

Descriptive statistics of the trial participants are reported in Table 1 . More than $65 \%$ of participants enrolled with a spouse as their study partner. Participants who enrolled with a spouse tended to be younger and were more likely to be male and non-Hispanic white when compared to those who enrolled with a non-spousal partner. Among global regions, similarly high proportions of spousal dyads were observed for North America, Western Europe/Israel, Australia/South Africa, Asia, and Japan. Higher proportions of non-spousal dyads were

Table 1 Sample description reported for all randomized participants with a reported study partner type. Quantitative variables are summarized as mean (standard deviation) and categorical variables as $N(\%)$

\begin{tabular}{|c|c|c|c|c|}
\hline & \multicolumn{3}{|l|}{ Study partner type } & \multirow{2}{*}{$\begin{array}{l}\text { Total }^{\mathrm{d}} \\
(N=2635)\end{array}$} \\
\hline & Spouse $(N=1729)$ & Adult child $(N=662)$ & Other $(N=244)$ & \\
\hline Participant age & $71.81(7.9)$ & $75.77(7.8)$ & $74.87(8.4)$ & $73.09(8.1)$ \\
\hline Education (years) & $13.07(3.9)$ & $10.61(4.1)$ & $11.13(4.1)$ & $12.27(4.1)$ \\
\hline Sex: female & $750(43 \%)$ & $519(78 \%)$ & $189(77 \%)$ & $1458(55 \%)$ \\
\hline \multicolumn{5}{|l|}{ Region $^{a}$} \\
\hline North America & $722(42 \%, 73 \%)$ & $172(26 \%, 17 \%)$ & $89(36 \%, 9 \%)$ & 983 (37\%) \\
\hline South America/Mexico & 87 (5\%, 43\%) & 77 (12\%, 38\%) & $39(16 \%, 19 \%)$ & $203(8 \%)$ \\
\hline Eastern Europe/Russia & $111(6 \%, 38 \%)$ & $153(23 \%, 53 \%)$ & $25(10 \%, 9 \%)$ & $289(11 \%)$ \\
\hline Western Europe/Israel & $413(24 \%, 75 \%)$ & $100(15 \%, 18 \%)$ & $36(15 \%, 7 \%)$ & $549(21 \%)$ \\
\hline Australia/South Africa & $113(7 \%, 74 \%)$ & $22(3 \%, 14 \%)$ & $18(7 \%, 12 \%)$ & $153(6 \%)$ \\
\hline Asia & $112(6 \%, 55 \%)$ & $74(11 \%, 36 \%)$ & $18(7 \%, 9 \%)$ & $204(8 \%)$ \\
\hline Japan & $171(10 \%, 67 \%)$ & $64(10 \%, 25 \%)$ & $19(8 \%, 7 \%)$ & $254(10 \%)$ \\
\hline \multicolumn{5}{|l|}{ Race/ethnicity } \\
\hline Caucasian & 1364 (79\%) & $452(68 \%)$ & $174(71 \%)$ & $1990(76 \%)$ \\
\hline Asian & $292(17 \%)$ & $142(21 \%)$ & $40(16 \%)$ & $474(18 \%)$ \\
\hline Hispanic & $57(3 \%)$ & $59(9 \%)$ & $24(10 \%)$ & $140(5 \%)$ \\
\hline African American/Black & $16(1 \%)$ & $9(1 \%)$ & $6(2 \%)$ & $31(1 \%)$ \\
\hline \multicolumn{5}{|l|}{ Baseline assessments } \\
\hline Mini-Mental State Exam & $20.94(3.2)$ & $20.06(3.0)$ & $20.66(3.0)$ & $20.69(3.1)$ \\
\hline ADAS-cog & $23.06(9.0)$ & $24.7(9.0)$ & $23.16(8.7)$ & $23.48(9.0)$ \\
\hline Activities of Daily Living & $61.08(12.2)$ & $57.42(14.5)$ & $58.72(14.6)$ & $59.95(13.1)$ \\
\hline Hachinski Score ${ }^{b}$ & $1(0,1)$ & $1(0,1)$ & $1(0,1)$ & $1(0,1)$ \\
\hline Geriatric Depression Scale ${ }^{b}$ & $2(1,3)$ & $2(1,3)$ & $2(1,3)$ & $2(1,3)$ \\
\hline \multicolumn{5}{|l|}{ Study partner } \\
\hline Age & $70.56(8.7)$ & $48.08(9.0)$ & $56.02(14.5)$ & $63.57(13.7)$ \\
\hline Contribution level $\left.\right|^{b, c}$ & $5(5,5)$ & $4(3,5)$ & $4(3,5)$ & $5(4,5)$ \\
\hline Sex: female & $972(56 \%)$ & 457 (69\%) & $200(82 \%)$ & $1629(62 \%)$ \\
\hline Paid job & 385 (22\%) & $451(68 \%)$ & 119 (49\%) & $955(36 \%)$ \\
\hline
\end{tabular}

${ }^{a}$ (Column \%, Row \%) are reported for region

${ }^{\mathrm{b}}$ Summarized as median (25\%, 75\%)

${ }^{c}$ Contribution level among all caregivers is measured on a scale from 1 (1-20\%) to 5 (81-100\%)

${ }^{\mathrm{d}}$ Two participants were excluded for having a race of 'other' and one was excluded for missing partner type 
observed in Eastern Europe/Russia and South America/ Mexico.

Among spousal dyads with complete covariates, 569 (33\%) were lost to follow-up, 28 (2\%) died, 655 (38\%) were forced to halt participation due to sponsor decision, and 469 (27\%) completed the week 76 co-primary endpoints. Among adult child dyads, 235 (36\%) were lost to follow-up, 16 died (2\%), 288 (44\%) were forced to halt participation, and 119 (18\%) completed the study. Among other dyads, 79 (35\%) were lost to follow-up, 1 died (<1\%), 98 (44\%) were forced to stop, and 46 (21\%) completed. Dyad-specific Kaplan-Meier curves estimating the probability of early trial exit by time since randomization are presented in Fig. 2. In unadjusted models, we estimated that participants with an adult child study partner had a $23 \%(\mathrm{RR}=1.23$; $95 \% \mathrm{CI} 1.06$, $1.42 ; p=0.007$ ) higher risk of dropping out compared to those with a spousal study partner; other dyads were estimated to have a $8 \%(\mathrm{RR}=1.08 ; 95 \% \mathrm{CI} 0.86,1.36 ; p=$ 0.524) higher risk of dropping out compared to spousal dyads. Although we observed a relationship between study partner type and early study exit in the unadjusted model, this relationship was no longer significant after adjusting for potential confounders. We estimated that subjects who enrolled with an adult child study partner had only a $6 \%(\mathrm{RR}=1.06 ; 95 \%$ CI $0.79,1.43 ; p=0.698)$ higher risk and other partners had a $3 \%(R R=1.03$; $95 \%$ CI $0.75,1.40 ; p=0.863$ ) higher risk of dropping out compared to those who enrolled with a spousal study partner when adjusting for potential confounders (Table 2). Sensitivity analyses censoring subjects who died or limiting the analysis to participants in North
America and adjusting for race did not substantially alter our results (Tables 3 and 4).

When comparing the fully adjusted model, unadjusted model, and the 11 models with one adjustment variable, we found that adjusting solely for participant age almost completely attenuated the relationship between study partner type and the risk of early exit to the null (Fig. 3). In the models only adjusting for age, participants with adult child partners were estimated to have a $6 \%$ higher risk of trial incompletion $(\mathrm{RR}=1.06 ; 95 \% \mathrm{CI} 0.91,1.24$; $p=0.443)$ and other dyads were estimated to have a $2 \%$ lower risk of trial incompletion $(\mathrm{RR}=0.98 ; 95 \% \mathrm{CI} 0.78$, $1.24 ; p=0.886)$ when compared to participants with spousal partners. Age had a strong association with dropout even after adjusting for all other considered confounders (Fig. 3). Predictive analyses utilizing random forests estimated the variable importance for each covariate and ranged from 0.0285 to less than 0.0001 . Participant age was the most predictive of trial incompletion with a variable importance of 0.0285 , continent was the second most predictive with a variable importance of 0.0133 , and the third most predictive variable was MMSE with a variable importance of 0.0048 .

\section{Discussion}

In two multinational industry-sponsored phase III trials of a candidate disease-modifying therapy for $\mathrm{AD}$, we observed differences in the characteristics and behaviors of the study dyads when looking at unadjusted proportions. In several global regions, individuals with spousal study partners were overrepresented, compared to population estimates of caregivers. Participants with a spousal study

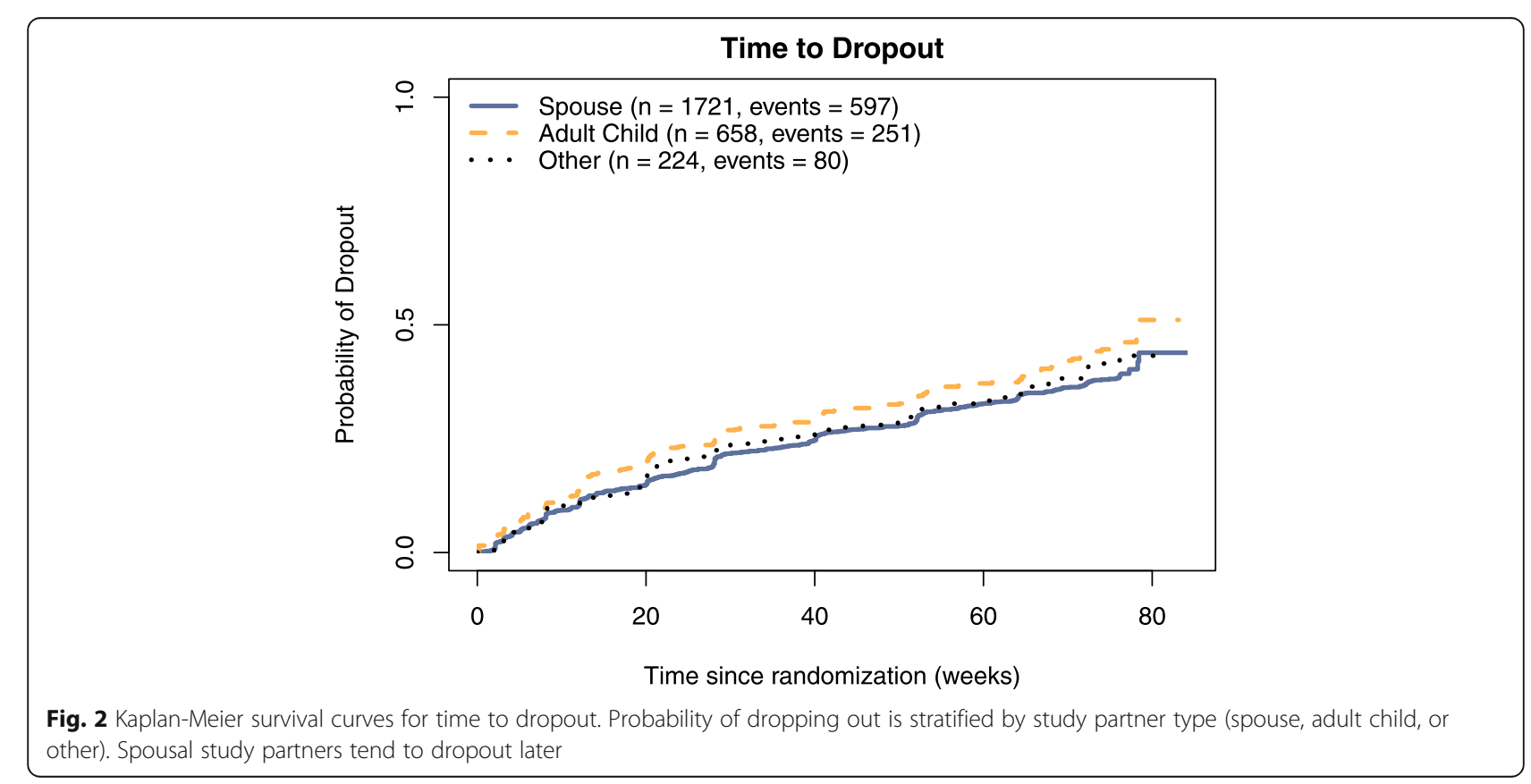


Table 2 Estimated relative risk (RR) for the risk of dropout

\begin{tabular}{|c|c|c|c|c|c|c|c|}
\hline & $\begin{array}{l}\text { Subjects } \\
(N=2603)\end{array}$ & $\begin{array}{l}\text { Events } \\
(N=928)\end{array}$ & $\begin{array}{l}\text { Deaths } \\
(N=45)\end{array}$ & Unadjusted RR & Unadj. $p$ value & Adjusted RR & Adj. $p$ value \\
\hline Partner type $^{a}$ & & & & & 0.027 & & 0.928 \\
\hline Spouse & 1721 & 597 & 28 & 1 & - & 1 & - \\
\hline Adult child & 658 & 251 & 16 & $1.23(1.06,1.42)$ & 0.007 & $1.06(0.79,1.43)$ & 0.698 \\
\hline Other & 224 & 80 & 1 & $1.08(0.86,1.36)$ & 0.524 & $1.03(0.75,1.40)$ & 0.863 \\
\hline Participant age (per 10 years) & 2603 & 928 & 45 & $1.41(1.29,1.53)$ & $<.001$ & $1.41(1.26,1.58)$ & $<.001$ \\
\hline Education (years) & 2603 & 928 & 45 & $0.98(0.96,1.00)$ & 0.011 & $0.99(0.97,1.01)$ & 0.165 \\
\hline Partner age (per 10 years) & 2603 & 928 & 45 & $1.04(0.99,1.09)$ & 0.15 & $1.02(0.93,1.13)$ & 0.645 \\
\hline \multicolumn{8}{|l|}{ Baseline test scores } \\
\hline Mini-Mental State Exam & 2603 & 928 & 45 & $0.94(0.92,0.96)$ & $<.001$ & $0.95(0.92,0.97)$ & $<.001$ \\
\hline Hachinski Score & 2603 & 928 & 45 & $1.14(1.06,1.22)$ & $<.001$ & $1.04(0.96,1.12)$ & 0.336 \\
\hline Geriatric Depression Scale & 2603 & 928 & 45 & $1.09(1.05,1.13)$ & $<.001$ & $1.09(1.05,1.14)$ & $<.001$ \\
\hline \multicolumn{8}{|l|}{ Participant sex } \\
\hline Female & 1437 & 493 & 19 & 1 & - & 1 & - \\
\hline Male & 1166 & 435 & 26 & $1.07(0.94,1.22)$ & 0.281 & $1.26(1.05,1.51)$ & 0.013 \\
\hline \multicolumn{8}{|l|}{ Partner sex } \\
\hline Female & 1602 & 588 & 26 & 1 & - & 1 & - \\
\hline Male & 1001 & 340 & 19 & $0.91(0.80,1.04)$ & 0.172 & $1.10(0.92,1.32)$ & 0.293 \\
\hline \multicolumn{8}{|l|}{ Partner employed } \\
\hline No & 1652 & 615 & 27 & 1 & - & 1 & - \\
\hline Yes & 951 & 313 & 18 & $0.88(0.77,1.01)$ & 0.06 & $0.93(0.79,1.09)$ & 0.373 \\
\hline \multicolumn{8}{|l|}{ APOE carrier } \\
\hline No & 903 & 333 & 20 & 1 & - & 1 & - \\
\hline Yes & 1700 & 595 & 25 & $0.94(0.82,1.08)$ & 0.396 & $1.00(0.87,1.15)$ & 0.993 \\
\hline \multicolumn{8}{|l|}{ Region } \\
\hline North America & 971 & 369 & 19 & 1 & - & 1 & - \\
\hline South America/Mexico & 201 & 81 & 6 & $1.24(0.98,1.57)$ & 0.08 & $1.03(0.79,1.34)$ & 0.828 \\
\hline Eastern Europe/Russia & 286 & 133 & 2 & $1.50(1.24,1.83)$ & $<.001$ & $1.44(1.16,1.80)$ & 0.001 \\
\hline Western Europe/Israel & 543 & 167 & 7 & $0.78(0.65,0.94)$ & 0.007 & $0.82(0.67,0.99)$ & 0.044 \\
\hline Australia/South Africa & 151 & 57 & 6 & $0.96(0.73,1.28)$ & 0.803 & $0.97(0.73,1.29)$ & 0.823 \\
\hline Asia & 200 & 62 & 2 & $1.07(0.82,1.40)$ & 0.608 & $1.02(0.77,1.35)$ & 0.899 \\
\hline Japan & 251 & 59 & 3 & $0.60(0.45,0.79)$ & $<.001$ & $0.58(0.44,0.78)$ & $<.001$ \\
\hline
\end{tabular}

${ }^{a}$ Partial likelihood ratio test of the null hypothesis that there is no difference in risk of dropping out by study partner (SP) type

partner were younger, more educated, more often male, and more often white than counterparts with nonspouse partners. They also dropped out of the trials less frequently than did participants with non-spousal partners. Overall, $35 \%$ of spousal dyad participants dropped out of the study or died, compared to $38 \%$ of participants with adult study partners and $36 \%$ of participants with other study partners. These results are consistent with previous observations in academic trials conducted exclusively in North America [10] and suggest that investigators may need to be alert to the increased risk of dropout for participants enrolling with a non-spouse partner.
Despite these observations in the raw data, multivariable analyses indicated that the increased risk of dropout among adult child dyads was not due to the study partner relationship, per se. Instead, the relationship between study partner type and dropout dissipated after controlling for participant age. This was true when the model outcome included or excluded participants lost due to death. Increased age may be a risk factor for dropout. In a previous study of $\mathrm{AD}$ trials, subjects were partitioned into age groups $(55-65,65-75$, and $>75)$ and higher rates of dropout and dropout due to adverse events in the older two groups were observed [25]. Our results conflict with previous studies, which found that 
Table 3 Sensitivity analysis - estimated relative risk (RR) for the risk of dropout when censoring participants who died

\begin{tabular}{|c|c|c|c|c|c|c|c|}
\hline & $\begin{array}{l}\text { Subjects } \\
(N=2603)\end{array}$ & $\begin{array}{l}\text { Events } \\
(N=928)\end{array}$ & $\begin{array}{l}\text { Deaths } \\
(N=45)\end{array}$ & Unadjusted RR & Unadj. $p$ value & Adjusted RR & Adj. $p$ value \\
\hline Partner type $^{a}$ & & & & & 0.57 & & 0.954 \\
\hline Spouse & 1721 & 569 & 28 & 1 & - & 1 & - \\
\hline Adult child & 658 & 235 & 16 & $1.20(1.03,1.40)$ & 0.018 & $1.03(0.76,1.39)$ & 0.837 \\
\hline Other & 224 & 79 & 1 & $1.12(0.88,1.41)$ & 0.356 & $1.05(0.77,1.44)$ & 0.756 \\
\hline Participant age (per 10 years) & 2603 & 883 & 45 & $1.37(1.26,1.49)$ & $<.001$ & $1.37(1.22,1.54)$ & $<.001$ \\
\hline Education (years) & 2603 & 883 & 45 & $0.98(0.97,1.00)$ & 0.016 & $0.99(0.97,1.01)$ & 0.205 \\
\hline Partner age (per 10 years) & 2603 & 883 & 45 & $1.04(0.98,1.09)$ & 0.188 & $1.02(0.93,1.13)$ & 0.623 \\
\hline \multicolumn{8}{|l|}{ Baseline test scores } \\
\hline Mini-Mental State Exam & 2603 & 883 & 45 & $0.94(0.92,0.96)$ & $<.001$ & $0.95(0.93,0.97)$ & $<.001$ \\
\hline Hachinski Score & 2603 & 883 & 45 & $1.12(1.05,1.21)$ & 0.001 & $1.02(0.95,1.10)$ & 0.586 \\
\hline Geriatric Depression Scale & 2603 & 883 & 45 & $1.10(1.05,1.14)$ & $<.001$ & $1.10(1.05,1.15)$ & $<.001$ \\
\hline \multicolumn{8}{|l|}{ Participant sex } \\
\hline Female & 1437 & 474 & 19 & 1 & - & 1 & - \\
\hline Male & 1166 & 409 & 26 & $1.05(0.92,1.20)$ & 0.471 & $1.18(0.98,1.42)$ & 0.085 \\
\hline \multicolumn{8}{|l|}{ Partner sex } \\
\hline Female & 1602 & 562 & 26 & 1 & - & 1 & - \\
\hline Male & 1001 & 321 & 19 & $0.90(0.78,1.03)$ & 0.135 & $1.04(0.86,1.25)$ & 0.684 \\
\hline \multicolumn{8}{|l|}{ Partner employed } \\
\hline No & 1652 & 588 & 27 & 1 & - & 1 & - \\
\hline Yes & 951 & 295 & 18 & $0.87(0.75,0.99)$ & 0.042 & $0.91(0.77,1.07)$ & 0.251 \\
\hline \multicolumn{8}{|l|}{ APOE carrier } \\
\hline No & 903 & 313 & 20 & 1 & - & 1 & - \\
\hline Yes & 1700 & 570 & 25 & $0.96(0.84,1.10)$ & 0.580 & $1.02(0.88,1.17)$ & 0.821 \\
\hline \multicolumn{8}{|l|}{ Region } \\
\hline North America & 971 & 350 & 19 & 1 & - & 1 & - \\
\hline South America/Mexico & 201 & 75 & 6 & $1.20(0.94,1.53)$ & 0.146 & $1.00(0.76,1.31)$ & 0.981 \\
\hline Eastern Europe/Russia & 286 & 131 & 2 & $1.55(1.27,1.89)$ & $<.001$ & $1.49(1.19,1.87)$ & $<.001$ \\
\hline Western Europe/Israel & 543 & 160 & 7 & $0.79(0.65,0.95)$ & 0.012 & $0.82(0.67,1.00)$ & 0.049 \\
\hline Australia/South Africa & 151 & 51 & 6 & $0.91(0.68,1.23)$ & 0.537 & $0.91(0.67,1.23)$ & 0.525 \\
\hline Asia & 200 & 60 & 2 & $1.08(0.82,1.42)$ & 0.567 & $1.03(0.77,1.37)$ & 0.854 \\
\hline Japan & 251 & 56 & 3 & $0.60(0.45,0.79)$ & $<.001$ & $0.58(0.43,0.78)$ & $<.001$ \\
\hline
\end{tabular}

Partial likelihood ratio test of the null hypothesis that there is no difference in risk of dropping out by study partner (SP) type

risk of dropout remained for non-spousal (other) dyads, even when controlling for age [10]. What factors may explain this discrepancy is unclear. The current trials were global, but restricting our analysis to participants from North America did not substantially alter results. The current trials were much larger, including a mix of academic and non-academic sites. These differences in trial design, size, and study population between the previous analysis and the current work may contribute to the differing conclusions. For example, previous analyses in similar large studies found that both participant marital status and site type were associated with participant retention $[5,14]$. We lacked information here to examine site type or a potential interaction between site and dyad types. Alternatively, we cannot rule out that other unmeasured differences between the study populations, such as research attitudes (Stites et al., in press), could have contributed the differences in observations.

The lack of significant relationship between dyad type and dropout (when controlling for covariates) may be good news for AD trial investigators. Increasing enrollment of participants with non-spousal partners represents a potentially key opportunity to address the crisis in trial recruitment [3]. Moreover, doing so may allow for more representative and generalizable study samples, given that non-spousal caregivers are most common [7- 
Table 4 Sensitivity analysis_estimated relative risk (RR) for the risk of dropout for participants in North America

\begin{tabular}{|c|c|c|c|c|c|c|c|}
\hline & $\begin{array}{l}\text { Subjects } \\
(N=2603)\end{array}$ & $\begin{array}{l}\text { Events } \\
(N=928)\end{array}$ & $\begin{array}{l}\text { Deaths } \\
(N=45)\end{array}$ & Unadjusted RR & Unadj. $p$ value & Adjusted RR & Adj. $p$ value \\
\hline Partner type $^{a}$ & & & & & 0.595 & & 0.53 \\
\hline Spouse & 720 & 268 & 13 & 1 & - & 1 & - \\
\hline Adult child & 171 & 69 & 6 & $1.13(0.86,1.48)$ & 0.374 & $0.81(0.49,1.36)$ & 0.427 \\
\hline Other & 80 & 32 & 0 & $1.12(0.78,1.62)$ & 0.532 & $1.06(0.66,1.72)$ & 0.802 \\
\hline Participant age (per 10 years) & 971 & 369 & 19 & $1.47(1.30,1.66)$ & $<.001$ & $1.47(1.23,1.75)$ & $<.001$ \\
\hline Education (years) & 971 & 369 & 19 & $0.95(0.92,0.98)$ & 0.001 & $0.97(0.93,1.00)$ & 0.034 \\
\hline Partner age (per 10 years) & 971 & 369 & 19 & $1.12(1.02,1.23)$ & 0.021 & $0.97(0.82,1.14)$ & 0.699 \\
\hline \multicolumn{8}{|l|}{ Baseline test scores } \\
\hline Mini-Mental State Exam & 971 & 369 & 19 & $0.94(0.91,0.97)$ & $<.001$ & $0.94(0.91,0.97)$ & $<.001$ \\
\hline Hachinski Score & 971 & 369 & 19 & $1.12(1.00,1.26)$ & 0.049 & $1.04(0.92,1.19)$ & 0.513 \\
\hline Geriatric Depression Scale & 971 & 369 & 19 & $1.08(1.01,1.15)$ & 0.035 & $1.08(1.01,1.16)$ & 0.031 \\
\hline \multicolumn{8}{|l|}{ Participant sex } \\
\hline Female & 504 & 188 & 8 & 1 & - & 1 & - \\
\hline Male & 467 & 181 & 11 & $1.02(0.83,1.26)$ & 0.821 & $1.02(0.73,1.42)$ & 0.903 \\
\hline \multicolumn{8}{|l|}{ Partner sex } \\
\hline Female & 600 & 239 & 13 & 1 & - & 1 & - \\
\hline Male & 371 & 130 & 6 & $0.86(0.69,1.06)$ & 0.162 & $0.88(0.64,1.23)$ & 0.469 \\
\hline \multicolumn{8}{|l|}{ Partner employed } \\
\hline No & 649 & 270 & 13 & 1 & - & 1 & - \\
\hline Yes & 322 & 99 & 6 & $0.69(0.54,0.86)$ & 0.001 & $0.75(0.57,0.99)$ & 0.046 \\
\hline \multicolumn{8}{|l|}{ APOE carrier } \\
\hline No & 318 & 127 & 7 & 1 & - & 1 & - \\
\hline Yes & 653 & 242 & 12 & $0.88(0.71,1.10)$ & 0.263 & $0.96(0.76,1.22)$ & 0.76 \\
\hline \multicolumn{8}{|l|}{ Race/ethnicity } \\
\hline White & 922 & 346 & 16 & 1 & - & 1 & - \\
\hline Asian & 13 & 6 & 0 & $1.13(0.54,2.38)$ & 0.741 & $1.15(0.50,2.63)$ & 0.743 \\
\hline Hispanic & 16 & 8 & 3 & $1.30(0.65,2.60)$ & 0.452 & $1.07(0.52,2.19)$ & 0.861 \\
\hline African American/Black & 20 & 9 & 0 & $1.43(0.70,2.93)$ & 0.325 & $1.18(0.52,2.68)$ & 0.69 \\
\hline
\end{tabular}

aPartial likelihood ratio test of the null hypothesis that there is no difference in risk of dropping out by study partner (SP) type

10] and are particularly prevalent among racial and ethnic communities that are even more drastically underrepresented in clinical trials $[6,26]$.

AD trials now enroll a spectrum of patient populations, including those with mild cognitive impairment ("prodromal AD" if biomarker evidence of $\mathrm{AD}$ is present) and those with normal cognitive performance but biomarker evidence of disease ("preclinical AD") [27]. Despite these diagnostic differences, all AD trials require dyadic participation and it will be important to examine whether similar relationships between study partner type and recruitment and retention are observed in preclinical and prodromal AD. To our knowledge, this matter is not well studied, though natural history studies suggest that dyad type may be similarly important in retention in predementia trials $[28,29]$.
To reduce missing data and subsequent bias, dropout must be minimized to the greatest extent possible. These results suggest that older participant age is a risk factor for this event. In previous meta-analytic studies, older participants in ADRD trials also demonstrated less decline on trial cognitive and functional outcome measures, potentially reducing trial power for demonstrating diseaseslowing effects [30, 31]. Older participants may therefore bring added risks to trial integrity. Most AD patients, however, are over age 75 [7]. Recent NIH policies aim to ensure inclusion of older more representative samples in research, especially clinical trials [32]. Trialists may need to consider optimal means for retaining older participants to trial completion. In particular, efforts to reduce the burden of participation and incentivize trial completion should be pursued, including financial support throughout 


\section{Adult Child Dyads (adult child vs. spouse)}

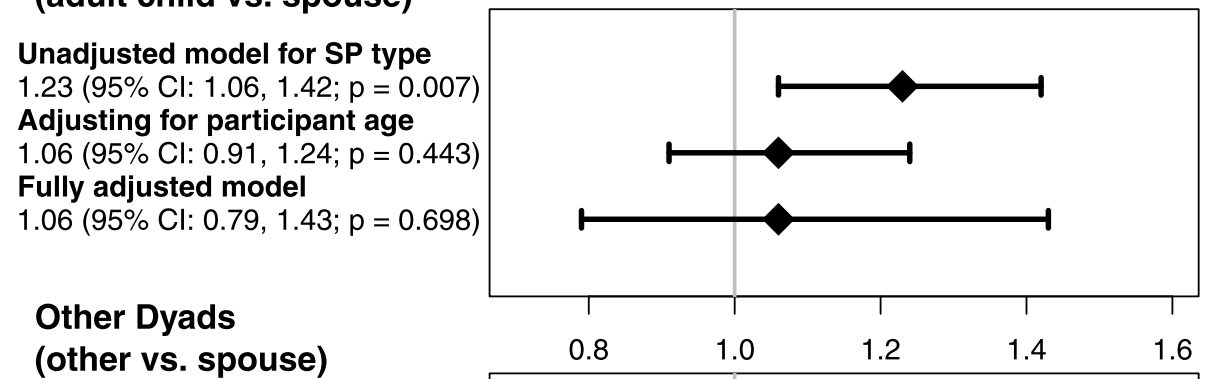

Unadjusted model for SP type

1.08 (95\% Cl: 0.86, 1.36; $p=0.524)$

Adjusting for participant age

$0.98(95 \% \mathrm{Cl}: 0.78,1.24 ; p=0.886)$

Fully adjusted model

$1.03(95 \% \mathrm{Cl}: 0.75,1.40 ; \mathrm{p}=0.863)$

Participant Age

(per 10 year increase)

Unadjusted model for age

1.41 (95\% Cl: 1.29, 1.53; $p=<.001)$

Adjusting for SP type

$1.40(95 \% \mathrm{Cl}: 1.28,1.52 ; \mathrm{p}=<.001)$

Fully adjusted model

$1.41(95 \% \mathrm{Cl}: 1.26,1.58 ; \mathrm{p}=<.001)$
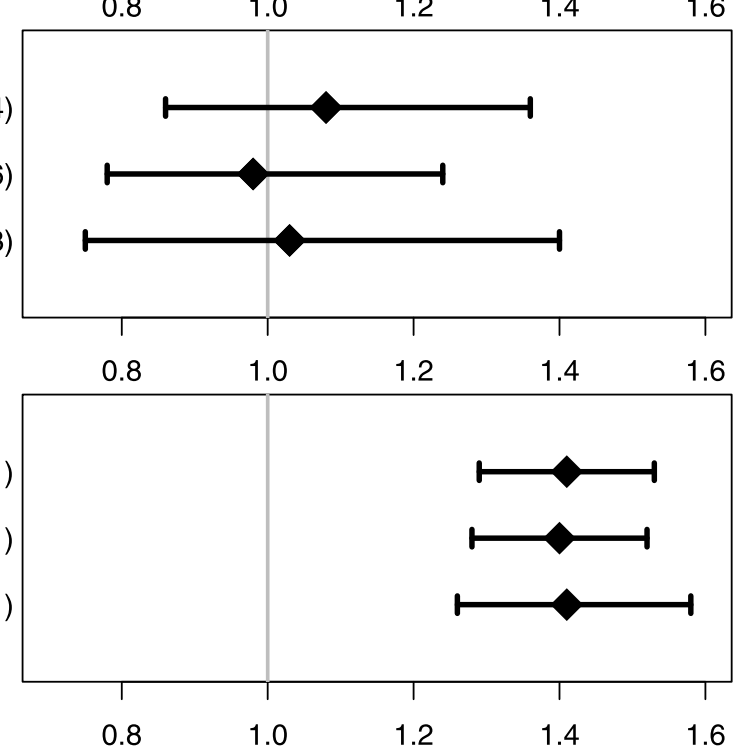

Relative Risk of Dropout

Fig. 3 Forest plots of the estimated relative risk of dropping out comparing adult child dyads to spousal dyads (top panel) and other dyads to spousal dyads (middle panel). The bottom panel shows the estimated relative risk of dropping out comparing to subpopulations of participants differing in age by 10 years. Each of the three plots shows three estimates: an unadjusted model, a model adjusting only for study partner (SP) type or participant age, and a fully adjusted model containing all potential confounders. Notice that the relationship between study partner type and dropout is attenuated after adjusting for participant age, but the relationship between participant age and dropout remains after adjusting for study partner type and all potential confounders

the trial and unique payment schedules if they improve completion rates [33, 34]. Such strategies may be particularly valuable for hard to recruit participants, notably including older participants, non-spouse dyads, and minority races and ethnicities [35]. Numerous other tactics for retaining participants exist [36], though few have been tested rigorously in the setting of trials, let alone for potential effect modification by participant (or study partner) characteristics.

\section{Limitations}

This study had limitations. We are unable to establish a causal relationship between study dyad characteristics and dropout due to the observational nature of this study and the potential for unmeasured confounding factors. We, however, a priori hypothesized which variables would be potential confounders or were predictive of the response and accounted for any that were measured. Residual confounding may exist with respect to socioeconomic status, study partner education level, marital status, site type, study partner depression or caregiving burden, comorbidities, or attitudes toward research. Higher levels of closeness between caregivers and participants also may be related to study partner type and have been reported to be associated with slower functional and cognitive decline, fewer neuropsychiatric symptoms, lower care costs, and both better and worse health outcomes for the caregiver [37-40]. Racial and ethnic minorities were underrepresented in this study $[10,14]$, which may have led to bias in the estimated relationship between partner type and dropout. Lastly, a large number of participants were unable to complete participation in the trial due to the administrative decision of the sponsor to halt the 
study. These participants did not choose to drop out of the trial but did not complete the study. Since accrual patterns among these participants did not differ by dyad type (data not shown), this was unlikely to bias estimates.

\section{Conclusions}

Previous analyses of smaller academic AD trials found that participants who enrolled with a spousal study partner were less likely to dropout. We did not fully replicate these findings within two multinational phase III studies. We instead found that age was both a strong predictor of who dropped out and a confounder of observed differences between dyad types that largely explained the relationship between study partner type and the risk of study incompletion. Future AD clinical trials need to prioritize including and retaining nonspousal dyads and older participants to prevent missingness and create more generalizable trial results.

\section{Abbreviations}

AD: Alzheimer's disease; ADAS-cog: Alzheimer's Disease Assessment Scale for cognition; ADCS-ADL: Alzheimer's Disease Cooperative Study-Activities of Daily Living; APOE4: Apolipoprotein E ع4; Cl: Confidence interval; DSMB: Data and safety monitoring board; LRT: Partial likelihood ratio test; MMSE: MiniMental State Examination; MCI: Mild cognitive impairment; NIA: National Institute of Aging; NINCDS-ADRDA: National Institute of Neurological and Communicative Disorders and Stroke and the Alzheimer's Disease and Related Disorders Association; RR: Relative risk; RUD: Resource Utilization in Dementia; SP: Study partner

\section{Acknowledgements}

We would like to thank the participants and study partners of these studies. Data for this study were provided by a data transfer agreement through the Alzheimer's Disease Cooperative Study (U19AG010483) and Eli Lilly.

\section{Authors' contributions}

OMB performed the analyses, interpreted the results, and drafted the manuscript. JDG and DLG secured the funding, designed the study, interpreted the results, and edited the manuscript for content. All authors approved the final manuscript.

\section{Funding}

This work was supported by NIH/NIA 1RF1AG059407. OMB is also supported by the National Science Foundation Graduate Research Fellowship under Grant No. DGE-183928. JDG is also supported by NIA AG066519 and NCATS UL1 TR001414.

\section{Availability of data and materials}

The data that support the findings of this study are available from Eli Lilly but restrictions apply to the availability of these data, which were used under license for the current study, and so are not publicly available. Data are however available from the authors upon reasonable request and with permission of Eli Lilly.

\section{Ethics approval and consent to participate}

This study used deidentified patient data and did not require ethics approval.

\section{Consent for publication}

Not applicable

\section{Competing interests}

JDG reports research support from Biogen, Eli Lilly, Genentech, the Alzheimer's Disease Cooperative Study, and the Alzheimer's Clinical Trial Consortium. He has no other competing interests.

\section{Author details}

'Department of Statistics, University of California, Irvine, Bren Hall 2019, Irvine, CA 92697-1250, USA. ${ }^{2}$ Institute for Memory Impairments and Neurological Disorders, University of California, Irvine, Irvine, CA, USA. ${ }^{3}$ Alzheimer's Disease Research Center, University of California, Irvine, Irvine, CA, USA. ${ }^{4}$ Department of Psychiatry and Human Behavior, University of California, Irvine, Irvine, CA, USA. ${ }^{5}$ Department of Neurobiology and Behavior, University of California, Irvine, Irvine, CA, USA.

Received: 25 October 2020 Accepted: 23 December 2020

Published online: 08 January 2021

\section{References}

1. Cummings J, Lee G, Ritter A, Sabbagh M, Zhong K. Alzheimer's disease drug development pipeline: 2019. Alzheimers Dement Transl Res Clin Intervent. 2019;5:272-93.

2. Cummings J, Ritter A, Zhong K. Clinical trials for disease-modifying therapies in Alzheimer's disease: a primer, lessons learned, and a blueprint for the future. J Alzheimers Dis. 2018;64:S3-22.

3. Fargo KN, Carrillo MC, Weiner MW, Potter WZ, Khachaturian Z. The crisis in recruitment for clinical trials in Alzheimer's and dementia: an action plan for solutions. Alzheimers Dement. 2016:12:1113-5.

4. Watson JL, Ryan L, Silverberg N, Cahan V, Bernard MA. Obstacles and opportunities in Alzheimer's clinical trial recruitment. Health Aff. 2014;33 574-9.

5. Grill JD, Karlawish J. Addressing the challenges to successful recruitment and retention in Alzheimer's disease clinical trials. Alzheimers Res Ther. 2010; 2:34.

6. Faison WE, Schultz SK, Aerssens J, Alvidrez J, Anand R, Farrer LA, et al. Potential ethnic modifiers in the assessment and treatment of Alzheimer's disease: challenges for the future. Int Psychogeriatr. 2007;19:539-58 Cambridge University Press.

7. 2020 Alzheimer's disease facts and figures. Alzheimers Dement. 2020;16(3): 391-460.

8. Riffin C, Van Ness PH, Wolff JL, Fried T. Family and other unpaid caregivers and older adults with and without dementia and disability. J Am Geriatr Soc. 2017;65:1821-8.

9. National Poll on Healthy Aging. Dementia Caregivers: Juggling, Delaying and Looking Forward. [Internet]. Available from: http://www. healthyagingpoll.org/sites/default/files/2017-10/NPHA_Caregivers-ReportPROOF_101817_v2.pdf.

10. Grill JD, Raman R, Ernstrom K, Aisen P, Karlawish J. Effect of study partner on the conduct of Alzheimer disease clinical trials. Neurology. 2013;80:282-8.

11. National Research Council (US) Panel on Handling Missing Data in Clinical Trials. The prevention and treatment of missing data in clinical trials [Internet]. Washington (DC): National Academies Press (US); 2010. Available from: http://www.ncbi.nlm.nih.gov/books/NBK209904/.

12. Sheiner LB. Learning versus confirming in clinical drug development. Clin Pharmacol Ther. 1997;61:275-91.

13. Petersen RC, Thomas RG, Aisen PS, Mohs RC, Carrillo MC, Albert MS, et al. Randomized controlled trials in mild cognitive impairment: sources of variability. Neurology. 2017;88:1751-8.

14. Edland SD, Emond JA, Aisen PS, Petersen RC. NIA-funded Alzheimer centers are more efficient than commercial clinical recruitment sites for conducting secondary prevention trials of dementia. Alzheimer Dis Assoc Disord. 2010; 24:159-64.

15. Dording CM, Dalton ED, Pencina MJ, Fava M, Mischoulon D. Comparison of academic and nonacademic sites in multi-center clinical trials. J Clin Psychopharmacol. 2012;32:65-8.

16. Grill JD, Raman R, Ernstrom $K$, Aisen P, Dowsett SA, Chen Y-F, et al. Comparing recruitment, retention, and safety reporting among geographic regions in multinational Alzheimer's disease clinical trials. Alzheimers Res Ther. 2015;7:39.

17. Cummings JL, Atri A, Ballard C, Boneva N, Frölich L, Molinuevo JL, et al. Insights into globalization: comparison of patient characteristics and disease progression among geographic regions in a multinational Alzheimer's disease clinical program. Alzheimers Res Ther. 2018;10:116.

18. Doody RS, Raman R, Farlow M, Iwatsubo T, Vellas B, Joffe $S$, et al. A phase 3 trial of semagacestat for treatment of Alzheimer's disease. N Engl J Med. 2013;369:341-50 Massachusetts Medical Society. 
19. Lilly Halts Development of Semagacestat for Alzheimer's Disease Based on Preliminary Results of Phase III Clinical Trials [Internet]. Eli Lilly and Company. [cited 2020 May 4]. Available from: https://investor.lilly.com/newsreleases/news-release-details/lilly-halts-development-semagacestatalzheimers-disease-based.

20. Henley DB, Dowsett SA, Chen Y-F, Liu-Seifert H, Grill JD, Doody RS, et al. Alzheimer's disease progression by geographical region in a clinical trial setting. Alzheimers Res Ther. 2015;7:43.

21. Ishwaran H, Kogalur UB, Blackstone EH, Lauer MS. Random survival forests. Ann Appl Stat. 2008;2:841-60.

22. Ishwaran H, Kogalur UB. randomForestSRC: fast unified random forests for survival, regression, and classification (RF-SRC) [Internet]. 2020. Available from: https://CRAN.R-project.org/package=randomForestSRC.

23. Ishwaran H, Kogalur U. Random survival forests for R. R News. 2007;7:25-31.

24. R Core Team. R: a language and environment for statistical computing [Internet]. Vienna: R Foundation for Statistical Computing; 2019. Available from: https://www.R-project.org/.

25. Henley DB, Sundell KL, Sethuraman G, Schneider LS. Adverse events and dropouts in Alzheimer's disease studies: what can we learn? Alzheimers Dement J Alzheimers Assoc. 2015;11:24-31.

26. Shin J, Doraiswamy PM. Underrepresentation of African-Americans in Alzheimer's trials: a call for affirmative action. Front Aging Neurosci. 2016;8:123.

27. Nuño MM, Gillen DL, Dosanjh KK, Brook J, Elashoff D, Ringman JM, et al. Attitudes toward clinical trials across the Alzheimer's disease spectrum. Alz Res Ther. 2017:9:81.

28. Koss E, Peterson B, Fillenbaum GG. Determinants of attrition in a natural history study of Alzheimer disease. Alzheimer Dis Assoc Disord. 1999;13: 209-15.

29. Coley N, Gardette V, Toulza O, Gillette-Guyonnet S, Cantet C, Nourhashemi $\mathrm{F}$, et al. Predictive factors of attrition in a cohort of Alzheimer disease patients. The REAL.FR study. Neuroepidemiology. 2008;31:69-79.

30. Bernick C, Cummings J, Raman R, Sun X, Aisen P. Age and rate of cognitive decline in Alzheimer disease: implications for clinical trials. Arch Neurol. 2012;69:901-5.

31. Schneider LS, Kennedy RE, Wang G, Cutter GR. Differences in Alzheimer disease clinical trial outcomes based on age of the participants. Neurology. 2015;84:1121-7.

32. Bernard MA, Clayton JA, Lauer MS. Inclusion across the lifespan: NIH policy for clinical research. JAMA. 2018;320:1535-6.

33. Largent EA, Fernandez LH. Making the case for completion bonuses in clinical trials. Clin Trials. 2019;16:176-82.

34. Largent EA, Karlawish J, Grill JD. Study partners: essential collaborators in discovering treatments for Alzheimer's disease. Alzheimers Res Ther. 2018; 10:101.

35. Brewster $P$, Barnes L, Haan M, Johnson JK, Manly JJ, Nápoles AM, et al. Progress and future challenges in aging and diversity research in the United States. Alzheimers Dement. 2019;15:995-1003.

36. Robinson KA, Dinglas VD, Sukrithan V, Yalamanchilli R, Mendez-Tellez PA Dennison-Himmelfarb C, et al. Updated systematic review identifies substantial number of retention strategies: using more strategies retains more study participants. J Clin Epidemiol. 2015;68:1481-7.

37. Vernon EK, Cooley B, Rozum W, Rattinger GB, Behrens S, Matyi J, et al. Caregiver-care recipient relationship closeness is associated with neuropsychiatric symptoms in dementia. The American Journal of Geriatric Psychiatry. Elsevier. 2019;27:349-59.

38. Rattinger GB, Fauth EB, Behrens S, Sanders C, Schwartz S, Norton MC, et al. Closer caregiver and care-recipient relationships predict lower informal costs of dementia care: the Cache County Dementia Progression Study. Alzheimers Dement. 2016;12:917-24.

39. Fauth E, Hess K, Piercy K, Norton M, Corcoran C, Rabins P, et al. Caregivers' relationship closeness with the person with dementia predicts both positive and negative outcomes for caregivers' physical health and psychological well-being. Aging Ment Health. 2012;16:699-711.

40. Norton MC, Piercy KW, Rabins PV, Green RC, Breitner JCS, Ostbye T, et al. Caregiver-recipient closeness and symptom progression in Alzheimer disease. The Cache County Dementia Progression Study. J Gerontol B Psychol Sci Soc Sci. 2009;64:560-8.

\section{Publisher's Note}

Springer Nature remains neutral with regard to jurisdictional claims in published maps and institutional affiliations.

Ready to submit your research? Choose BMC and benefit from:

- fast, convenient online submission

- thorough peer review by experienced researchers in your field

- rapid publication on acceptance

- support for research data, including large and complex data types

- gold Open Access which fosters wider collaboration and increased citations

- maximum visibility for your research: over $100 \mathrm{M}$ website views per year

At BMC, research is always in progress.

Learn more biomedcentral.com/submissions 